\title{
Existence and nonexistence in the liquid drop model
}

\author{
Rupert L. Frank ${ }^{1,2,3}$ (D) Phan Thành Nam ${ }^{1,2}$
}

Received: 6 January 2021 / Accepted: 26 July 2021 / Published online: 16 September 2021

(c) The Author(s) 2021

\begin{abstract}
We revisit the liquid drop model with a general Riesz potential. Our new result is the existence of minimizers for the conjectured optimal range of parameters. We also prove a conditional uniqueness of minimizers and a nonexistence result for heavy nuclei.
\end{abstract}

Mathematics Subject Classification 49Q10 $\cdot 49$ Q20 $\cdot 81$ V35

\section{Introduction}

Let $N \geq 2, \lambda \in(0, N)$ and $m>0$ ( $\lambda$ and $m$ are not necessarily integers). For any measurable set $\Omega \subset \mathbb{R}^{N}$, define

$$
\mathcal{E}(\Omega)=\operatorname{Per} \Omega+D(\Omega), \quad D(\Omega)=\frac{1}{2} \iint_{\Omega \times \Omega} \frac{\mathrm{d} x \mathrm{~d} y}{|x-y|^{\lambda}} .
$$

The perimeter Per $\Omega$ is taken in the sense of De Giorgi, namely

$$
\text { Per } \Omega=\sup \left\{\int_{\Omega} \operatorname{div} F(x) \mathrm{d} x\left|F \in C_{0}^{1}\left(\mathbb{R}^{3}, \mathbb{R}^{3}\right),\right| F \mid \leq 1\right\},
$$

which is simply the surface area of $\Omega$ when the boundary is smooth. We consider the minimization problem

$$
E(m)=\inf _{|\Omega|=m} \mathcal{E}(\Omega)
$$

Communicated by E. Lenzmann.

This paper may be reproduced, in its entirety, for non-commercial purposes.

$\triangle$ Rupert L. Frank

rlfrank@caltech.edu

Phan Thành Nam

nam@math.lmu.de

1 Department of Mathematics, LMU Munich, Theresienstrasse 39, 80333 Munich, Germany

2 Munich Center for Quantum Science and Technology (MCQST), Schellingstr. 4, 80799 München, Germany

3 Department of Mathematics, California Institute of Technology, Pasadena, CA 91125, USA 
The most important case is $\lambda=1$ in dimension $N=3$, which goes back to Gamow's liquid drop model for atomic nuclei [15]. In this case, a nucleus is thought of consisting of nucleons (protons and neutrons) in a set $\Omega \subset \mathbb{R}^{N}$. The nucleons are assumed to be concentrated with constant density, which implies that the number of nucleons is proportional to $|\Omega|$. The perimeter term in the energy functional corresponds to a surface tension, which holds the nuclei together. The second term in the energy functional corresponds to a Coulomb repulsion among the protons. Here for simplicity we have scaled all physical constants to be unity.

In the last decade, this model (for general $\lambda$ and $N$ ) has gained renewed interest in the mathematics literature. We refer to [6] for a review and, for instance, to [2,9-12,16-19,22] and references therein; see also [14,25]. A variant of the problem with a constant background has also been intensely studied, see, for instance, $[1,3-5,8,13,20]$ and references therein.

In principle, the two terms in $\mathcal{E}(\Omega)$ are competing against each other: balls minimize the first term (by the isoperimetric inequality [7], see also [23, Theorem 14.1]) and maximize the second term (by the Riesz rearrangement inequality [26], see also [21, Theorem 3.7]). Thus the question about the existence of a minimizer for $E(m)$ is nontrivial.

Clearly, the existence will depend on the parameter $m>0$. By scaling $\Omega \mapsto m^{1 / N} U$ with $|U|=1$, we see that

$$
\mathcal{E}(\Omega)=m^{\frac{N-1}{N}} \operatorname{Per} U+m^{\frac{2 N-\lambda}{N}} D(U)=m^{\frac{N-1}{N}}\left(\operatorname{Per} U+m^{\frac{N+1-\lambda}{N}} D(U)\right) .
$$

Note that $(N+1-\lambda) / N>0$. This suggests that for small $m$ the short range attraction due to the perimeter term is dominant, whereas for large $m$ the long range repulsion due to the Riesz potential is dominant. Correspondingly, we expect that there is a minimizer for small $m$ and there is no minimizer for large $m$.

In the case $\lambda=1, N=3$, the physics literature suggests that there is a critical volume $m_{*}>0$ such that balls are unique minimizers for $E(m)$ when $m \leq m_{*}$ and there is no minimizer when $m>m_{*}$. The value $m_{*}$ corresponds to the threshold where the energy of a ball of volume $m$ is equal to that of two balls of mass $m / 2$, spaced infinitely far apart. It can be computed explicitly to be (see [5,12])

$$
m_{*}=\frac{\left|B_{1}\right| \operatorname{Per} B_{1}}{D\left(B_{1}\right)} \cdot \frac{2^{1 / 3}-1}{1-2^{-2 / 3}}=5 \frac{2^{1 / 3}-1}{1-2^{-2 / 3}} \approx 3.512
$$

with $B_{1}$ the unit ball in $\mathbb{R}^{3}$. A mathematical proof of this remains unknown.

In the present paper, we consider the general case $N \geq 2$ and $\lambda \in(0, N)$. We define the critical volume $m_{*}$ to be the unique value such that

$$
\mathcal{E}\left(\left(\frac{m_{*}}{\left|B_{1}\right|}\right)^{\frac{1}{N}} B_{1}\right)=2 \mathcal{E}\left(\left(\frac{m_{*}}{2\left|B_{1}\right|}\right)^{1 / N} B_{1}\right),
$$

namely,

$$
m_{*}=\left(\frac{2^{1 / N}-1}{1-2^{(\lambda-N) / N}} \cdot \frac{\operatorname{Per} B_{1}}{D\left(B_{1}\right)}\right)^{N /(N+1-\lambda)}\left|B_{1}\right| .
$$

Here $B_{1}$ is the unit ball in $\mathbb{R}^{N}$ (hence, $\left(m /\left|B_{1}\right|\right)^{1 / N} B_{1}$ is a ball of measure $m$ ). Thus, just like in the special case $\lambda=1, N=3$, this is the critical value where the energy of a ball of volume $m_{*}$ is equal to that of two balls of mass $m_{*} / 2$, spaced infinitely far apart, and it is natural to conjecture that $m_{*}$ divides the regime where minimizers are balls from the regime where there are no minimizers.

The following results were proved by Knüpfer and Muratov [18,19]: 
(a) For every $N \geq 2$ and $\lambda \in(0, N)$, there exists a constant $m_{c_{1}}>0$ such that $E(m)$ has a minimizer for every $m \leq m_{c_{1}}$.

(b) For every $N \geq 2$ and $\lambda \in(0,2)$, there exists a constant $m_{c_{2}}>0$ such that $E(m)$ has no minimizer for every $m>m_{c_{2}}$.

(c) If $N=2$ and $\lambda>0$ is sufficiently small, then $m_{c_{1}}=m_{c_{2}}=m_{*}$ and balls are unique minimizers for $E(m)$ with $m \leq m_{*}$.

(d) if $N=2$ and $\lambda<2$, or if $3 \leq N \leq 7$ and $\lambda<N-1$, then there exists a constant $0<m_{c_{1}}^{\prime} \leq m_{c_{1}}$ such that balls are unique minimizers for $E(m)$ with $m<m_{c_{1}}^{\prime}$.

In the most important case $\lambda=1, N=3$, see also [11,22] for alternative proofs of the non-existence result (b) and [16] for a short proof of the uniqueness result (d). In [24], Muratov and Zaleski proved (c) for the explicit range $0<\lambda \leq 0.034$ and $N=2$. In [2], Bonacini and Cristoferi extended (c) and (d) to all $N \geq 2$. In [9], Figalli, Fusco, Maggi, Millot and Morini extended (d) to all $N \geq 2$ and $\lambda \in(0, N)$.

Our first new result concerns the existence in (a). Except when $\lambda>0$ is small, the existence of minimizers for $E(m)$ is known only for small $m$. In this paper, we extend the existence to what is conjectured to be the optimal range of parameters.

Theorem 1 Let $N \geq 2$ and $\lambda \in(0, N)$. Then the variational problem $E(m)$ has a minimizer for every $0<m \leq m_{*}$, where $m_{*}$ is defined in (1).

We will prove Theorem 1 by establishing the strict binding inequality [12]

$$
E(m)<E\left(m_{1}\right)+E\left(m-m_{1}\right), \quad \forall 0<m_{1}<m
$$

for all $m<m_{*}$. As a by product of our proof, we obtain the following conditional uniqueness of minimizers.

Theorem 2 Let $N \geq 2$ and $\lambda \in(0, N)$. If $E(m)$ has no minimizer when $m>m_{*}$, then balls are minimizers for $E(m)$ when $m \leq m_{*}$ and they are unique minimizers when $m<m_{*}$.

So far, the non-existence result in the sharp range $m>m_{*}$ is only available for $\lambda>0$ small $[2,18,24]$. For larger $\lambda$ and a nonexplicit range of $m$, we have

Theorem 3 Let $N \geq 2$ and $\lambda \in(0, N)$ and $\lambda \leq 2$. Then there exists a constant $m_{c_{2}} \geq m_{*}$ such that $E(m)$ does not have a minimizer for all $m>m_{c_{2}}$.

This result is due to $[18,19,22]$ for $\lambda<2$ and seems to be unpublished for $\lambda=2$. We will combine the methods in [11] and [18,19]. It is an open problem whether the nonexistence result also holds for $2<\lambda<N$ when $N \geq 3$.

\section{Existence}

In this section we prove Theorem 1 . We will deduce Theorem 1 from the following strict binding inequality.

Theorem 4 Let $N \geq 2$ and $\lambda \in(0, N)$. Then for every $0<m<m_{*}$ with $m_{*}$ in (1), we have

$$
E(m)<E\left(m_{1}\right)+E\left(m-m_{1}\right), \quad \forall 0<m_{1}<m .
$$

Thanks to [12, Theorem 3.1], the strict binding inequality (3) is a sufficient condition for the existence of minimizers of $E(m)$. Moreover, by [12, Theorem 3.4], the set $\{m>$ $0: E(m)$ has a minimizer $\}$ is closed in $(0, \infty)$. Hence, Theorem 4 implies the existence of 
minimizers of $E(m)$ for all $0<m \leq m_{*}$. Note that the proofs of Theorems 3.1 and 3.4 in [12] extend, without modifications, to the case $\lambda \neq 1$; see Remark 3.7 in that paper.

We will prove the strict binding inequality using a scaling argument, based on the following key observation which uses only the isoperimetric inequality.

Lemma 5 If $0<m_{1}<m$, then we have, with $s=m_{1} / m \in(0,1)$ and $B_{1}$ the unit ball in $\mathbb{R}^{N}$,

$$
E\left(m_{1}\right) \geq s^{(2 N-\lambda) / N} E(m)+\left(1-s^{(N+1-\lambda) / N}\right) s^{(N-1) / N}\left(\frac{m}{\left|B_{1}\right|}\right)^{(N-1) / N} \operatorname{Per} B_{1} .
$$

Proof Take $\Omega \subset \mathbb{R}^{N}$ such that $|\Omega|=m_{1}$. Then $\left|s^{-1 / N} \Omega\right|=m$, and hence

$$
\begin{aligned}
E(m) \leq \mathcal{E}\left(s^{-1 / N} \Omega\right) & =s^{-(N-1) / N} \operatorname{Per} \Omega+s^{-(2 N-\lambda) / N} D(\Omega) \\
& =s^{-(2 N-\lambda) / N} \mathcal{E}(\Omega)-\left(s^{-(2 N-\lambda) / N}-s^{-(N-1) / N}\right) \operatorname{Per} \Omega .
\end{aligned}
$$

By the isoperimetric inequality

$$
\text { Per } \Omega \geq\left(\frac{m_{1}}{\left|B_{1}\right|}\right)^{(N-1) / N} \operatorname{Per} B_{1}=s^{(N-1) / N}\left(\frac{m}{\left|B_{1}\right|}\right)^{(N-1) / N} \operatorname{Per} B_{1} .
$$

Thus

$$
E(m) \leq s^{-(2 N-\lambda) / N} \mathcal{E}(\Omega)-\left(s^{-(2 N-\lambda) / N}-s^{-(N-1) / N}\right) s^{(N-1) / N}\left(\frac{m}{\left|B_{1}\right|}\right)^{(N-1) / N} \operatorname{Per} B_{1} .
$$

Optimizing over all $\Omega$ satisfying $|\Omega|=m_{1}$ we get

$$
E(m) \leq s^{-(2 N-\lambda) / N} E\left(m_{1}\right)-\left(s^{-(2 N-\lambda) / N}-s^{-(N-1) / N}\right) s^{(N-1) / N}\left(\frac{m}{\left|B_{1}\right|}\right)^{(N-1) / N} \operatorname{Per} B_{1}
$$

which is equivalent to the desired inequality.

Proof of Theorem 4 Take $0<m_{1}<m<m_{*}$. Denote $s=m_{1} / m \in(0,1)$. By Lemma 5 we have

$$
\begin{aligned}
E\left(m_{1}\right) \geq & s^{(2 N-\lambda) / N} E(m)+\left(1-s^{(N+1-\lambda) / N}\right) s^{(N-1) / N}\left(\frac{m}{\left|B_{1}\right|}\right)^{(N-1) / N} \operatorname{Per} B_{1}, \\
E\left(m-m_{1}\right) \geq & (1-s)^{(2 N-\lambda) / N} E(m) \\
& +\left(1-(1-s)^{(N+1-\lambda) / N}\right)(1-s)^{(N-1) / N}\left(\frac{m}{\left|B_{1}\right|}\right)^{(N-1) / N} \operatorname{Per} B_{1} .
\end{aligned}
$$

Therefore,

$$
\begin{aligned}
& E\left(m_{1}\right)+E\left(m-m_{1}\right)-E(m) \geq\left(s^{(2 N-\lambda) / N}+(1-s)^{(2 N-\lambda) / N}-1\right) E(m) \\
& +\left(\left(1-s^{(N+1-\lambda) / N}\right) s^{(N-1) / N}+\left(1-(1-s)^{(N+1-\lambda) / N}\right)(1-s)^{(N-1) / N}\right) \\
& \times\left(\frac{m}{\left|B_{1}\right|}\right)^{(N-1) / N} \text { Per } B_{1} .
\end{aligned}
$$

Moreover, by the variational principle,

$$
E(m) \leq \mathcal{E}\left(\left(\frac{m}{\left|B_{1}\right|}\right)^{1 / N} B_{1}\right)=\left(\frac{m}{|B|}\right)^{(N-1) / N} \operatorname{Per} B_{1}+\left(\frac{m}{\left|B_{1}\right|}\right)^{(2 N-\lambda) / N} D\left(B_{1}\right) .
$$


Inserting (5) in (4) and using

$$
s^{(2 N-\lambda) / N}+(1-s)^{(2 N-\lambda) / N}-1<0, \quad \forall s \in(0,1),
$$

we find that

$$
\begin{aligned}
E( & \left.m_{1}\right)+E\left(m-m_{1}\right)-E(m) \\
\geq & \left(s^{(2 N-\lambda) / N}+(1-s)^{(2 N-\lambda) / N}-1\right)\left(\left(\frac{m}{|B|}\right)^{(N-1) / N} \operatorname{Per} B_{1}+\left(\frac{m}{\left|B_{1}\right|}\right)^{(2 N-\lambda) / N} D\left(B_{1}\right)\right) \\
& +\left(\left(1-s^{(N+1-\lambda) / N}\right) s^{(N-1) / N}+\left(1-(1-s)^{(N+1-\lambda) / N}\right)(1-s)^{(N-1) / N}\right) \\
& \times\left(\frac{m}{\left|B_{1}\right|}\right)^{(N-1) / N} \operatorname{Per} B_{1} \\
= & \left(s^{(N-1) / N}+(1-s)^{(N-1) / N}-1\right)\left(\frac{m}{\left|B_{1}\right|}\right)^{(N-1) / N} \operatorname{Per} B_{1} \\
& +\left(s^{(2 N-\lambda) / N}+(1-s)^{(2 N-\lambda) / N}-1\right)\left(\frac{m}{\left|B_{1}\right|}\right)^{(2 N-\lambda) / N} D\left(B_{1}\right) \\
= & \left(s^{(2 N-\lambda) / N}+(1-s)^{(2 N-\lambda) / N}-1\right)\left(\frac{m}{\left|B_{1}\right|}\right)^{(N-1) / N} \operatorname{Per} B_{1} \\
& \times\left(\frac{D\left(B_{1}\right)}{\operatorname{Per} B_{1}}\left(\frac{m}{\left|B_{1}\right|}\right)^{(N+1-\lambda) / N}-f(s)\right)
\end{aligned}
$$

with

$$
f(s):=\frac{s^{(N-1) / N}+(1-s)^{(N-1) / N}-1}{1-s^{(2 N-\lambda) / N}-(1-s)^{(2 N-\lambda) / N}} .
$$

Using again (6), we find that the strict binding inequality

$$
E\left(m_{1}\right)+E\left(m-m_{1}\right)-E(m)>0
$$

holds true if

$$
f(s)>\frac{D\left(B_{1}\right)}{\operatorname{Per} B_{1}}\left(\frac{m}{\left|B_{1}\right|}\right)^{(N+1-\lambda) / N}, \quad \forall s \in(0,1) .
$$

On the other hand, we can show that (see Lemma 6 below)

$$
\min _{s \in(0,1)} f(s)=f(1 / 2)=\frac{2^{1 / N}-1}{1-2^{(\lambda-N) / N}} .
$$

Therefore, (8) holds true when

$$
\frac{2^{1 / N}-1}{1-2^{(\lambda-N) / N}}>\frac{D\left(B_{1}\right)}{\operatorname{Per} B_{1}}\left(\frac{m}{\left|B_{1}\right|}\right)^{(N+1-\lambda) / N}
$$

which is equivalent to $m<m_{*}$.

It remains to prove (9). We have

Lemma 6 For all $0<a<1<b<2$ and $0<s<1$ we have

$$
\frac{s^{a}+(1-s)^{a}-1}{2^{1-a}-1} \geq-\frac{s \log s+(1-s) \log (1-s)}{\log 2} \geq \frac{s^{b}+(1-s)^{b}-1}{2^{1-b}-1} .
$$

The inequality (9) follows from Lemma 6 with $a=(N-1) / N$ and $b=(2 N-\lambda) / N$. 

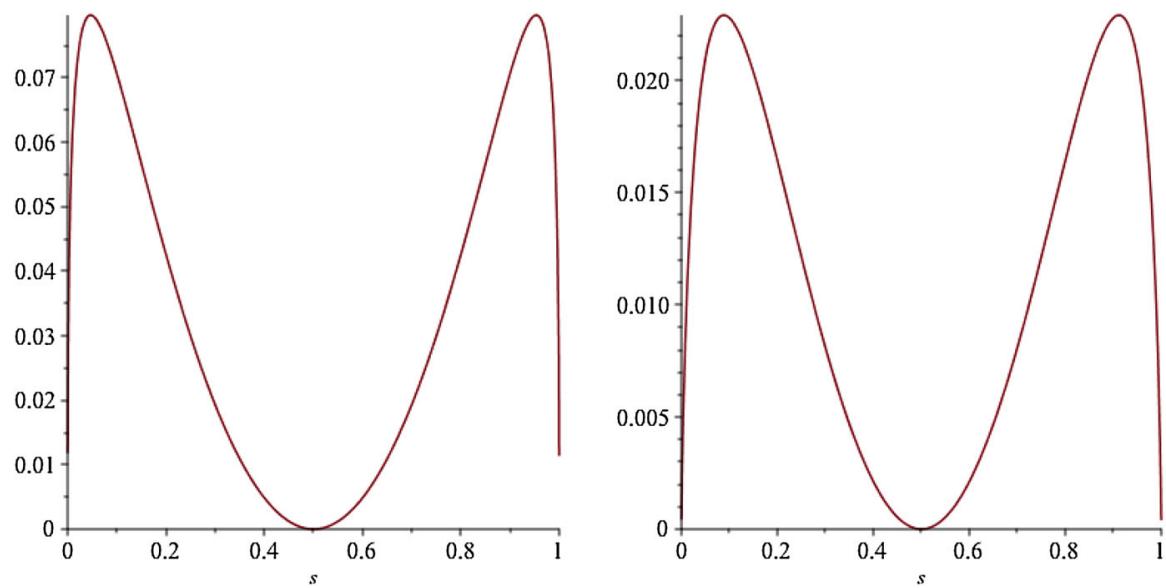

Fig. 1 The function $g(s), s \in(0,1)$, with $\alpha=0.5$ (left) and $\alpha=1.5$ (right)

Proof We will prove that for all $\alpha \in(0,2)$ and all $s \in(0,1)$,

$$
g(s):=s^{\alpha}+(1-s)^{\alpha}-1+\frac{2^{1-\alpha}-1}{\log 2}(s \log s+(1-s) \log (1-s)) \geq 0 .
$$

Then the desired conclusion follows from (10) and the fact that $2^{1-\alpha}-1>0$ if $\alpha \in(0,1)$ while $2^{1-\alpha}-1<0$ if $\alpha \in(1,2)$ (Fig. 1).

By the symmetry $s \leftrightarrow 1-s$, it suffices to prove (10) for $s \in(0,1 / 2]$. Also, (10) is trivial when $\alpha=1$, so we will distinguish two cases $\alpha \in(0,1)$ and $\alpha \in(1,2)$.

Case 1: $\alpha \in(0,1)$. We have

$$
\begin{aligned}
& g^{\prime}(s)=\alpha\left(s^{\alpha-1}-(1-s)^{\alpha-1}\right)+\frac{2^{1-\alpha}-1}{\log 2}(\log s-\log (1-s)), \\
& g^{\prime \prime}(s)=\alpha(\alpha-1)\left(s^{\alpha-2}+(1-s)^{\alpha-2}\right)+\frac{2^{1-\alpha}-1}{\log 2}\left(s^{-1}+(1-s)^{-1}\right) .
\end{aligned}
$$

Define $h:(0,1 / 2] \rightarrow \mathbb{R}$ by

$$
\begin{aligned}
h(s) & :=s(1-s) g^{\prime \prime}(s) \\
& =\alpha(\alpha-1) s(1-s)\left(s^{\alpha-2}+(1-s)^{\alpha-2}\right)+\frac{2^{1-\alpha}-1}{\log 2} \\
& =\alpha(\alpha-1)\left(s^{\alpha-1}+(1-s)^{\alpha-1}-s^{\alpha}-(1-s)^{\alpha}\right)+\frac{2^{1-\alpha}-1}{\log 2} .
\end{aligned}
$$

Note that for all $s \in(0,1 / 2)$ we have

$$
h^{\prime}(s)=\alpha(\alpha-1)^{2}\left(s^{\alpha-2}-(1-s)^{\alpha-2}\right)+\alpha^{2}(1-\alpha)\left(s^{\alpha-1}-(1-s)^{\alpha-1}\right)>0
$$

since

$$
s^{\alpha-2}-(1-s)^{\alpha-2}>0, \quad(1-\alpha)\left(s^{\alpha-1}-(1-s)^{\alpha-1}\right)>0 .
$$

Thus $h$ is strictly increasing on $(0,1 / 2]$. Moreover,

$$
\lim _{s \rightarrow 0^{+}} h(s)=-\infty
$$


and

$$
\begin{aligned}
h(1 / 2) & =\alpha(\alpha-1) 2^{1-\alpha}+\frac{2^{1-\alpha}-1}{\log 2}=2^{1-\alpha}\left(\frac{1}{\log 2}-\alpha(1-\alpha)\right)-\frac{1}{\log 2} \\
& \geq(1+(1-\alpha) \log 2)\left(\frac{1}{\log 2}-\alpha(1-\alpha)\right)-\frac{1}{\log 2} \\
& =(1-\alpha)^{2}[1-\alpha \log 2]>0,
\end{aligned}
$$

since

$$
2^{1-\alpha}=e^{(1-\alpha) \log 2} \geq 1+(1-\alpha) \log 2, \quad \alpha(1-\alpha) \leq \frac{1}{4}<\frac{1}{\log 2} .
$$

Thus there exists a unique value $s_{1} \in(0,1 / 2)$ (depending on $\alpha$ ) such that

$$
h(s)<0 \text { on } s \in\left(0, s_{1}\right), \quad h(s)>0 \text { on } s \in\left(s_{1}, 1 / 2\right) .
$$

Putting back the definition $h(s)=s(1-s) g^{\prime \prime}(s)$, we find that

$$
g^{\prime \prime}(s)<0 \text { on } s \in\left(0, s_{1}\right), \quad g^{\prime \prime}(s)>0 \text { on } s \in\left(s_{1}, 1 / 2\right) .
$$

Thus $g^{\prime}(s)$ is strictly decreasing on $s \in\left(0, s_{1}\right)$ and strictly increasing on $s \in\left(s_{1}, 1 / 2\right)$. Combining with

$$
\lim _{s \rightarrow 0^{+}} g^{\prime}(s)=\infty, \quad g^{\prime}(1 / 2)=0,
$$

we find that there exists a unique value $s_{2} \in(0,1 / 2)$ (depending on $\alpha$ ) such that

$$
g^{\prime}(s)>0 \text { on } s \in\left(0, s_{2}\right), \quad g^{\prime}(s)<0 \text { on } s \in\left(s_{2}, 1 / 2\right) .
$$

Thus $g(s)$ is strictly increasing on $s \in\left(0, s_{2}\right)$ and strictly decreasing on $s \in\left(s_{2}, 1 / 2\right)$. Therefore,

$$
\inf _{s \in(0,1 / 2]} g(s)=\min \left\{\lim _{s \rightarrow 0^{+}} g(s), g(1 / 2)\right\}=0 .
$$

Case 2: $\alpha \in(1,2)$. We can proceed similarly. To be precise, the function $h(s)=s(1-s) g^{\prime \prime}(s)$ also satisfies

$$
h^{\prime}(s)=\alpha(\alpha-1)^{2}\left(s^{\alpha-2}-(1-s)^{\alpha-2}\right)+\alpha^{2}(1-\alpha)\left(s^{\alpha-1}-(1-s)^{\alpha-1}\right)>0
$$

for all $s \in(0,1 / 2)$. Thus $h$ is also strictly increasing on $(0,1 / 2]$. Moreover,

$$
\lim _{s \rightarrow 0^{+}} h(s)=\frac{2^{1-\alpha}-1}{\log 2}<0
$$

and

$$
\begin{aligned}
h(1 / 2) & =\alpha(\alpha-1) 2^{1-\alpha}+\frac{2^{1-\alpha}-1}{\log 2}=\alpha(\alpha-1) 2^{1-\alpha}+\frac{e^{(1-\alpha) \log 2}-1}{\log 2} \\
& \geq \alpha(\alpha-1) 2^{1-\alpha}+1-\alpha=(\alpha-1)\left(\alpha 2^{1-\alpha}-1\right)>0 .
\end{aligned}
$$

Here we have used $\alpha 2^{1-\alpha}>1$ for all $\alpha \in(1,2)$ (the function $q(\alpha)=\alpha 2^{1-\alpha}$ is concave on $(1,2)$ as $q^{\prime \prime}(\alpha)=2^{1-\alpha}(\log 2)(\alpha \log 2-2)<0$ and $\left.q(1)=q(2)=1\right)$.

Thus there exists a unique value $s_{1} \in(0,1 / 2)$ (depending on $\alpha$ ) such that

$$
h(s)<0 \text { on } s \in\left(0, s_{1}\right), \quad h(s)>0 \text { on } s \in\left(s_{1}, 1 / 2\right) .
$$

The rest is exactly the same as in Case 1 . This completes the proof of Lemma 6. 


\section{Uniqueness}

Proof of Theorem 2 Step 1 We prove that balls are minimizers for $E\left(m_{*}\right)$. Assume by contradiction that balls are not minimizers for $E\left(m_{*}\right)$, namely

$$
E\left(m_{*}\right)<\mathcal{E}\left(\left(m_{*} /\left|B_{1}\right|\right)^{1 / N} B_{1}\right) .
$$

Since $m \mapsto E(m)$ and $m \mapsto \mathcal{E}\left(\left(m /\left|B_{1}\right|\right)^{1 / N} B_{1}\right)$ are continuous (for the first function, see [12, Proof of Theorem 3.1]), there exists a constant $\delta \in(0,1)$ such that for all $m \in\left[m_{*}, m_{*}+\delta\right)$ we have

$$
\begin{aligned}
E(m) & \leq(1-\delta) \mathcal{E}\left(\left(m /\left|B_{1}\right|\right)^{1 / N} B_{1}\right) \\
& \leq\left(\frac{m}{\left|B_{1}\right|}\right)^{(N-1) / N} \operatorname{Per} B_{1}+\left(\frac{m}{\left|B_{1}\right|}\right)^{(2 N-\lambda) / N}(1-\delta) D\left(B_{1}\right) .
\end{aligned}
$$

This is similar to $(5)$, but $D\left(B_{1}\right)$ is replaced by $(1-\delta) D\left(B_{1}\right)$. Proceeding similarly as in the proof of Theorem 4 and inserting (11) (instead of (5)) in (4), for all $m \in\left[m_{*}, m_{*}+\delta\right.$ ) and $0<m_{1}<m$ we have

$$
\begin{aligned}
& E\left(m_{1}\right)+E\left(m-m_{1}\right)-E(m) \\
& \geq\left(s^{(2 N-\lambda) / N}+(1-s)^{(2 N-\lambda) / N}-1\right)\left(\frac{m}{\left|B_{1}\right|}\right)^{(N-1) / N} \text { Per } B_{1} \times \\
& \quad \times\left(\frac{(1-\delta) D\left(B_{1}\right)}{\operatorname{Per} B_{1}}\left(\frac{m}{\left|B_{1}\right|}\right)^{(N+1-\lambda) / N}-f(s)\right)
\end{aligned}
$$

with $s=m_{1} / m \in(0,1)$ and with the same function $f(s)$ in (7). By (9), we conclude that

$$
E\left(m_{1}\right)+E\left(m-m_{1}\right)-E(m)>0, \quad \forall 0<m_{1}<m
$$

provided that

$$
\frac{2^{1 / N}-1}{1-2^{(\lambda-N) / N}} \geq \frac{(1-\delta) D\left(B_{1}\right)}{\operatorname{Per} B_{1}}\left(\frac{m}{\left|B_{1}\right|}\right)^{(N+1-\lambda) / N}
$$

which is equivalent to

$$
m \leq m_{*}(1-\delta)^{-N /(N+1-\lambda)} .
$$

Thus the variational problem $E(m)$ has a minimizer for all

$$
m \leq \min \left\{m_{*}+\delta, m_{*}(1-\delta)^{-N /(N+1-\lambda)}\right\} .
$$

This is a contradiction to the assumption that $E(m)$ has no minimizer if $m>m_{*}$. Thus we conclude that balls are minimizers for $E\left(m_{*}\right)$.

Step 2 Now we prove that if $m<m_{*}$, then balls are unique minimizers for $E(m)$. This fact follows from [2, Theorem 2.10] which states that the set where balls are minimizers is an interval and that one has uniqueness away from the endpoint (note that this part does not require the assumption $\lambda<N-1$ which is imposed in the rest of [2]). For the reader's convenience, we provide a direct proof below.

Consider an arbitrary measurable set $\Omega \subset \mathbb{R}^{N}$ with $|\Omega|=m<m_{*}$. Then proceeding as in the proof of Lemma 5, we find that

$$
\mathcal{E}(\Omega) \geq s^{(2 N-\lambda) / N} E\left(m_{*}\right)+\left(1-s^{(N+1-\lambda) / N}\right) s^{(N-1) / N}\left(\frac{m_{*}}{\left|B_{1}\right|}\right)^{(N-1) / N} \operatorname{Per} B_{1}
$$


with $s=m / m_{*} \in(0,1)$ and the equality occurs if and only if $\Omega$ is a ball. On the other hand, we know that balls are minimizers for $E\left(m_{*}\right)$, namely

$$
E\left(m_{*}\right)=\mathcal{E}\left(\left(\frac{m_{*}}{\left|B_{1}\right|}\right)^{1 / N} B_{1}\right)=\left(\frac{m_{*}}{\left|B_{1}\right|}\right)^{(N-1) / N} \operatorname{Per} B_{1}+\left(\frac{m_{*}}{\left|B_{1}\right|}\right)^{(2 N-\lambda) / N} D\left(B_{1}\right) .
$$

Inserting the latter equality in (12), we obtain

$$
\begin{aligned}
\mathcal{E}(\Omega) \geq & s^{(2 N-\lambda) / N}\left(\left(\frac{m_{*}}{|B|}\right)^{(N-1) / N} \operatorname{Per} B_{1}+\left(\frac{m_{*}}{\left|B_{1}\right|}\right)^{(2 N-\lambda) / N} D\left(B_{1}\right)\right) \\
& +\left(1-s^{(N+1-\lambda) / N}\right) s^{(N-1) / N}\left(\frac{m_{*}}{\left|B_{1}\right|}\right)^{(N-1) / N} \operatorname{Per} B_{1} \\
= & \left(\frac{m}{\left|B_{1}\right|}\right)^{(N-1) / N} \operatorname{Per} B_{1}+\left(\frac{m}{\left|B_{1}\right|}\right)^{(2 N-\lambda) / N} D\left(B_{1}\right)=\mathcal{E}\left(\left(\frac{m}{B_{1}}\right)^{1 / N} B_{1}\right) .
\end{aligned}
$$

Thus balls are minimizers for $E(m)$; moreover, if $\Omega$ is a minimizer for $E(m)$, then the equality occurs in (12) and $\Omega$ is a ball.

\section{Nonexistence}

In this section we prove Theorem 3 . First, by extending the analysis for $\lambda=1$ in [11] to general $\lambda$, we have

Lemma 7 Let $N \geq 2$ and $\lambda \in(0, N)$. Let $m>0$ be arbitrary. Let $\Omega \subset \mathbb{R}^{N}$ be a minimizer for $E(m)$. Then

$$
\iint_{\Omega \times \Omega} \frac{d x d y}{|x-y|^{\lambda-1}} \lesssim|\Omega|
$$

with an implied constant depending only on $\lambda$ and $N$.

It is unclear to us whether the power $\lambda-1$ in Lemma 7 is optimal. If we could replace this power by a smaller one, then we would be able to improve the condition $\lambda \leq 2$ in Theorem 3 .

Proof For $v \in \mathbb{S}^{N-1}$ and $t \in \mathbb{R}$ we set

$$
\Omega_{v, t}^{ \pm}:=\Omega \cap\left\{x \in \mathbb{R}^{N}: \pm v \cdot x> \pm t\right\} .
$$

For any $\rho \geq 0$, the set

$$
\Omega_{v, t}^{+} \cup\left(\Omega_{v, t}^{-}-\rho v\right)
$$

has measure $\left|\Omega_{v, t}^{+} \cup\left(\Omega_{v, t}^{-}-\rho v\right)\right|=|\Omega|=m$ and therefore, by minimality of $\Omega$,

$$
\mathcal{E}\left(\Omega_{\nu, t}^{+} \cup\left(\Omega_{v, t}^{-}-\rho v\right)\right) \geq \mathcal{E}(\Omega)
$$

For any $\rho>0$, we have

$$
\operatorname{Per}\left(\Omega_{v, t}^{+} \cup\left(\Omega_{v, t}^{-}-\rho v\right)\right)=\operatorname{Per} \Omega_{v, t}^{+}+\operatorname{Per} \Omega_{v, t}^{-} \leq \operatorname{Per} \Omega+2 \sigma(\Omega \cap\{v \cdot x=t\}),
$$

where $\sigma$ denotes the induced measure on the hyperplane $\{v \cdot x=t\}$ and where the inequality holds for almost every $t \in \mathbb{R}$.

On the other hand, for any $\rho \geq 0$,

$$
\iint_{\left(\Omega_{v, t}^{+} \cup\left(\Omega_{v, t}^{-}-\rho v\right)\right) \times\left(\Omega_{v, t}^{+} \cup\left(\Omega_{v, t}^{-}-\rho v\right)\right)} \frac{d x d y}{|x-y|^{\lambda}}=\iint_{\Omega_{v, t}^{+} \times \Omega_{v, t}^{+}} \frac{d x d y}{|x-y|^{\lambda}}+\iint_{\Omega_{v, t}^{-} \times \Omega_{v, t}^{-}} \frac{d x d y}{|x-y|^{\lambda}}
$$




$$
+2 \iint_{\Omega_{v, t}^{+} \times \Omega_{v, t}^{-}} \frac{d x d y}{|x-y+\rho \nu|^{\lambda}} .
$$

The last double integral tends to zero as $\rho \rightarrow \infty$

Inserting these facts into (13) and letting $\rho \rightarrow \infty$, we infer

$$
\begin{aligned}
& \operatorname{Per} \Omega+2 \sigma(\Omega \cap\{v \cdot x=t\})+\frac{1}{2} \iint_{\Omega_{v, t}^{+} \times \Omega_{v, t}^{+}} \frac{d x d y}{|x-y|^{\lambda}}+\frac{1}{2} \iint_{\Omega_{v, t}^{-} \times \Omega_{v, t}^{-}} \frac{d x d y}{|x-y|^{\lambda}} \\
& \quad \geq \mathcal{E}(\Omega) \\
& \quad=\operatorname{Per} \Omega+\frac{1}{2} \iint_{\Omega_{v, t}^{+} \times \Omega_{v, t}^{+}} \frac{d x d y}{|x-y|^{\lambda}}+\frac{1}{2} \iint_{\Omega_{v, t}^{-} \times \Omega_{v, t}^{-}} \frac{d x d y}{|x-y|^{\lambda}}+\iint_{\Omega_{v, t}^{+} \times \Omega_{v, t}^{-}} \frac{d x d y}{|x-y|^{\lambda}},
\end{aligned}
$$

that is,

$$
\sigma(\Omega \cap\{v \cdot x=t\}) \geq \frac{1}{2} \iint_{\Omega_{v, t}^{+} \times \Omega_{v, t}^{-}} \frac{d x d y}{|x-y|^{\lambda}} .
$$

Note that the double integral here can be written as $\iint_{\Omega \times \Omega}|x-y|^{-\lambda} \mathbb{1}_{\{v \cdot x>t>v \cdot y\}} d x d y$. Thus, integrating the inequality with respect to $t \in \mathbb{R}$ gives, by Fubini's theorem,

$$
|\Omega| \geq \frac{1}{2} \iint_{\Omega \times \Omega} \frac{(v \cdot(x-y))_{+}}{|x-y|^{\lambda}} d x d y .
$$

Finally, we average this inequality with respect to $v \in \mathbb{S}^{N-1}$ and use the fact that

$$
\int_{\mathbb{S}^{N-1}}(v \cdot(x-y))_{+} \frac{d v}{\left|\mathbb{S}^{N-1}\right|}=c_{N}|x-y|,
$$

to obtain the bound in the lemma.

With Lemma 7 at hand, it is easy to finish the proof of Theorem 3 if $\lambda \leq 1$. In fact, if $\lambda=1$, the lemma gives directly $|\Omega|^{2} \lesssim|\Omega|$, which is the claimed bound. If $0<\lambda<1$, by Riesz's rearrangement inequality we have for all $t>0$

$$
\iint_{\Omega \times \Omega} t^{-1}\left(1-e^{-t|x-y|^{1-\lambda}}\right) d x d y \geq \iint_{\Omega^{*} \times \Omega^{*}} t^{-1}\left(1-e^{-t|x-y|^{1-\lambda}}\right) d x d y,
$$

where $\Omega^{*}$ is the ball centered at 0 with volume $\left|\Omega^{*}\right|=|\Omega|$. Taking $t \rightarrow 0$ we obtain

$$
\iint_{\Omega \times \Omega} \frac{d x d y}{|x-y|^{\lambda-1}} \geq \iint_{\Omega^{*} \times \Omega^{*}} \frac{d x d y}{|x-y|^{\lambda-1}} \gtrsim\left|\Omega^{*}\right|^{(2 N-\lambda+1) / N}=|\Omega|^{(2 N-\lambda+1) / N} .
$$

Since $(2 N-\lambda+1) / N>1$, the lemma implies once again $|\Omega| \lesssim 1$.

It remains to deal with the case $1<\lambda \leq 2$. The key is the following bound, which in the special case $N=3$ and $\lambda=1$ appears in [22, Eq. (2.12)]. The proof there extends immediately to the general case, since the analogues of [22, Lemma 3 (ii) and Lemma 4] hold according to [19, Lemmas 4.1 and 4.3].

Lemma 8 Let $N \geq 2$ and $\lambda \in(0, N)$. Let $m \geq \omega_{N}$ and let $\Omega \subset \mathbb{R}^{N}$ be a minimizer for $E(m)$. Then, for $1 \leq R \leq \operatorname{diam} \Omega$,

$$
\left|\Omega \cap B_{R}(x)\right| \gtrsim R \quad \text { for a.e. } x \in \Omega,
$$

with an implied constant depending only on $\lambda$ and $N$. 
Here diam $\Omega$ in the lemma is understood as the diameter of the set $\left\{x \in \mathbb{R}^{N}:\left|\Omega \cap B_{r}(x)\right|>\right.$ 0 for all $r>0$.

We will use this lemma to deduce Theorem 3 for $1<\lambda \leq 2$. If diam $\Omega \leq 2$, then $|\Omega| \lesssim 1$ and we are done. Thus, assuming $\operatorname{diam} \Omega>2$, we have, by Lemma 8 ,

$$
\begin{aligned}
\iint_{\Omega \times \Omega} \frac{d x d y}{|x-y|^{\lambda-1}} & =(\lambda-1) \int_{0}^{\infty} \frac{d R}{R^{\lambda}}|\{(x, y) \in \Omega \times \Omega:|x-y|<R\}| \\
& =(\lambda-1) \int_{0}^{\infty} \frac{d R}{R^{\lambda}} \int_{\Omega} d x\left|\Omega \cap B_{R}(x)\right| \\
& \geq(\lambda-1) \int_{1}^{\operatorname{diam} \Omega} \frac{d R}{R^{\lambda}} \int_{\Omega} d x\left|\Omega \cap B_{R}(x)\right| \\
& \gtrsim \int_{1}^{\operatorname{diam} \Omega} \frac{d R}{R^{\lambda-1}}|\Omega| .
\end{aligned}
$$

The right side is bounded from below by a constant times $|\Omega|(\operatorname{diam} \Omega)^{2-\lambda}$ if $\lambda<2$ and by a constant times $|\Omega| \log \operatorname{diam} \Omega$ if $\lambda=2$. Combining this lower bound on the double integral with the upper bound from Lemma 7, we infer in either case that diam $\Omega \lesssim 1$, which implies $|\Omega| \lesssim 1$ and therefore concludes the proof of Theorem 3 .

Acknowledgements Partial support through U.S. National Science Foundation grants DMS-1363432 and DMS-1954995 (R.L.F.) and through the Deutsche Forschungsgemeinschaft (DFG, German Research Foundation) through Germany's Excellence Strategy EXC - 2111 - 390814868 (R.L.F., P.T.N.) is acknowledged.

Funding Open Access funding enabled and organized by Projekt DEAL.

Open Access This article is licensed under a Creative Commons Attribution 4.0 International License, which permits use, sharing, adaptation, distribution and reproduction in any medium or format, as long as you give appropriate credit to the original author(s) and the source, provide a link to the Creative Commons licence, and indicate if changes were made. The images or other third party material in this article are included in the article's Creative Commons licence, unless indicated otherwise in a credit line to the material. If material is not included in the article's Creative Commons licence and your intended use is not permitted by statutory regulation or exceeds the permitted use, you will need to obtain permission directly from the copyright holder. To view a copy of this licence, visit http://creativecommons.org/licenses/by/4.0/.

\section{References}

1. Alberti, G., Choksi, R., Otto, F.: Uniform energy distribution for an isoperimetric problem with long-range interactions. J. Am. Math. Soc. 22, 596-605 (2009)

2. Bonacini, M., Cristoferi, R.: Local and global minimality results for a nonlocal isoperimetric problem on $\mathbb{R}^{N}$. SIAM J. Math. Anal. 46(4), 2310-2349 (2014)

3. Cicalese, M., Spadaro, E.: Droplet minimizers of an isoperimetric problem with long-range interactions. Commun. Pure Appl. Math. 66, 1298-1333 (2013)

4. Choksi, R., Peletier, M.A.: Small volume fraction limit of the diblock copolymer problem: I. Sharpinterface functional. SIAM J. Math. Anal. 42, 1334-1370 (2010)

5. Choksi, R., Peletier, M.A.: Small volume-fraction limit of the diblock copolymer problem: II. Diffuseinterface functional. SIAM J. Math. Anal. 43(2), 739-763 (2011)

6. Choksi, R., Muratov, C. B., Topaloglu, I.: An Old Problem Resurfaces Nonlocally: Gamow's Liquid Drops Inspire Today's Research and Applications, Notices of the AMS (2017)

7. De Giorgi, E.: Sulla proprietà isoperimetrica dell'ipersfera, nella classe degli insiemi aventi frontiera orientata di misura finita. Atti Accad. Naz. Lincei. Mem. Cl. Sci. Fis. Mat. Nat. Sez. I(8), 33-44 (1958)

8. Emmert, L., Frank, R.L., König, T.: Liquid drop model for nuclear matter in the dilute limit. SIAM J. Math. Anal. 52(2), 1980-1999 (2020)

9. Figalli, A., Fusco, N., Maggi, F., Millot, V., Morini, M.: Isoperimetry and stability properties of balls with respect to nonlocal energies. Commun. Math. Phys. 336, 441-507 (2015) 
10. Frank, R.L.: Non-spherical equilibrium shapes in the liquid drop model. J. Math. Phys. 60, 071506 (2019)

11. Frank, R.L., Killip, R., Nam, P.T.: Nonexistence of large nuclei in the liquid drop model. Lett. Math. Phys. 106, 1033-1036 (2016)

12. Frank, R.L., Lieb, E.H.: A compactness lemma and its application to the existence of minimizers for the liquid drop model. SIAM J. Math. Anal. 47(6), 4436-4450 (2015)

13. Frank, R.L., Lieb, E.H.: Periodic energy minimizers for a one-dimensional liquid drop model. Lett. Math. Phys. 109(9), 2069-2081 (2019)

14. Frank, R.L., Nam, P.T., Van Den Bosch, H.: The ionization conjecture in Thomas-Fermi-Dirac-von Weizsäcker theory. Commun. Pure Appl. Math. 71(3), 577-614 (2018)

15. Gamow, G.: Mass defect curve and nuclear constitution. Proc. R. Soc. Lond. Ser. A 126, 632-644 (1930)

16. Julin, V.: Isoperimetric problem with a Coulombic repulsive term. Indiana Univ. Math. J. 63, 77-89 (2014)

17. Julin, V.: Remark on a nonlocal isoperimetric problem. Nonlinear Anal. 154, 174-188 (2017)

18. Knüpfer, H., Muratov, C.: On an isoperimetric problem with a competing nonlocal term I. The planar case. Commun. Pure Appl. Math. 66, 1129-1162 (2013)

19. Knüpfer, H., Muratov, C.: On an isoperimetric problem with a competing nonlocal term II. The general case. Commun. Pure Appl. Math. 67, 1974-1994 (2014)

20. Knüpfer, H., Muratov, C., Novaga, M.: Low density phases in a uniformly charged liquid. Commun. Math. Phys. 345, 141-183 (2016)

21. Lieb, E.H., Loss, M.: Analysis. Graduate Studies in Mathematics, 2nd edn., p. 14. American Mathematical Society, Providence, RI (2001)

22. Lu, J., Otto, F.: Nonexistence of minimizers for Thomas-Fermi-Dirac-von Weizsäcker model. Commun. Pure Appl. Math. 67(10), 1605-1617 (2014)

23. Maggi, F.: Sets of finite perimeter and geometric variational problems. Cambridge Studies in Advanced Mathematics, 135. Cambridge Univ. Press, Cambridge (2012)

24. Muratov, C., Zaleski, A.: On an isoperimetric problem with a competing non-local term: quantitative results. Ann. Global Anal. Geom. 47, 63-80 (2014)

25. Nam, P.T.: The Ionization Problem. EMS Newsl. 12, 22-27 (2020)

26. Riesz, F.: Sur une inégalité intégrale. J. Lond. Math. Soc. 5, 162-168 (1930)

Publisher's Note Springer Nature remains neutral with regard to jurisdictional claims in published maps and institutional affiliations. 